\title{
33
}

\section{The Chandrasekhar mass of a gravitating electron crystal}

\author{
D. ENGELHARDT AND I. BUES \\ Dr. Remeis Sternwarte, 96049 Bamberg, Germany
}

\begin{abstract}
The internal structure of a white dwarf may be changed by a strong magnetic field. A local model of the electrons is constructed within a thermal density matrix formalism, essentially a Heisenberg magnetism model. This results in a matrix Fermi function which is used to construct an isothermal model of the electron crystal. The central density of the crystal is $10^{8} \mathrm{~kg} / \mathrm{m}^{3}$ independent of the magnetic field within the plasma and therefore lower than the relativistic density, whereas this density is constant until the Fermi momentum $x f=0.3 * m_{e} * c$. Chandrasekhar masses up to $1.44 * 1.4 M_{0}$ are possible for polarizations of the plasma zone lower than 0.5 , if the temperature is close to the Curie point, whereas the crystal itself destabilizes the white dwarf depenclent on temperature.
\end{abstract}

\subsection{Introduction}

From the theory of magnetic phase transitions of solid state physics (Grosse 1988 ) it is expected, that the structure of a single white dwarf is changed drastically by a magnetic field. The polarized electrons throughout the star may interact due to a magnetic field. The nonlinear influence of a crystallization transition and the crystal itself may change the mass and radius of a white dwarf. We construct a thermal Heisenberg model of the electrons which results in a Fermi matrix function, which predicts a plasma crystal phase transition. This Fermi function is used within the standard white dwarf theory. An additional magnetic field stabilizes the star if the white dwarf is not totally crystallized. 


\subsection{The local model}

The quantum mechanical state of an electron is determined by the symmetry of the magnetic field. The electron state with polarization may be written in terms of density matrices $\rho$, e.g.:

$$
\rho=\sum_{i} \Psi_{e}^{i} * \Psi_{e}^{i+}
$$

where $\Psi_{e}$ represents a four component spinor solution of the Dirac equation, summed over all degenerate states $i$. The spinors are transformed with a Lorentz transformation. The density matrix represents a kinetic probability distribution. An interaction $\mu$ exists, which we assume to be proportional to $\rho$, e.g. $\mu \propto \rho$. Thus the complete Hamiltonian may be written as

$$
H=\sum_{i} E_{i} \rho+V_{i} \mu
$$

where $E_{i}$ and $V_{i}$ represent numerical values of the kinetic and the interaction energy of the ith electron, respectively. This form is similar to the Heisenberg operator. The weighted mean of the energy of many electrons is taken in terms of a Boltzmann distribution $w$ under periodic boundary conditions,

$$
w=\exp (-\beta H),
$$

where $\beta$ is the inverse temperature $T$. The expectation value of the energy is calculated as

$$
<E>=\frac{\rho E}{\exp (\beta H)+1},
$$

which represents a Fermi matrix function. For $\beta \rightarrow \infty(\mathrm{T}=0 \mathrm{~K})$ and $H<0$ as well as for $\beta \rightarrow 0 T \rightarrow \infty$ the energy is proportional to $\rho$, e.g. $E \propto \rho$, which represents the Maxwellian limit of a polarized nondegenerate gas. For $H>0$ and $\beta \rightarrow \infty(T \rightarrow 0)$ the electrons crystallize, since

$$
<E>\propto \rho * \exp (-\beta H) \propto \lim _{x \rightarrow \infty} \rho^{x} .
$$

Our model is restricted to $4 \times 4$ matrices of degenerate electrons, thus the basic assumptions are valid for metallic hydrogen. The more realistic situation of a carbon or Fe crystal may be also be described by (5), since the model is a three dimensional lattice model. The matrix function is calculated by the use of the Jones (1941) calculus, which uses the fact that the function of a matrix is similar to the matrix itself. This concept was used succesfully for the calculation of the matrix Planck function and leads to the Bose - Einstein 


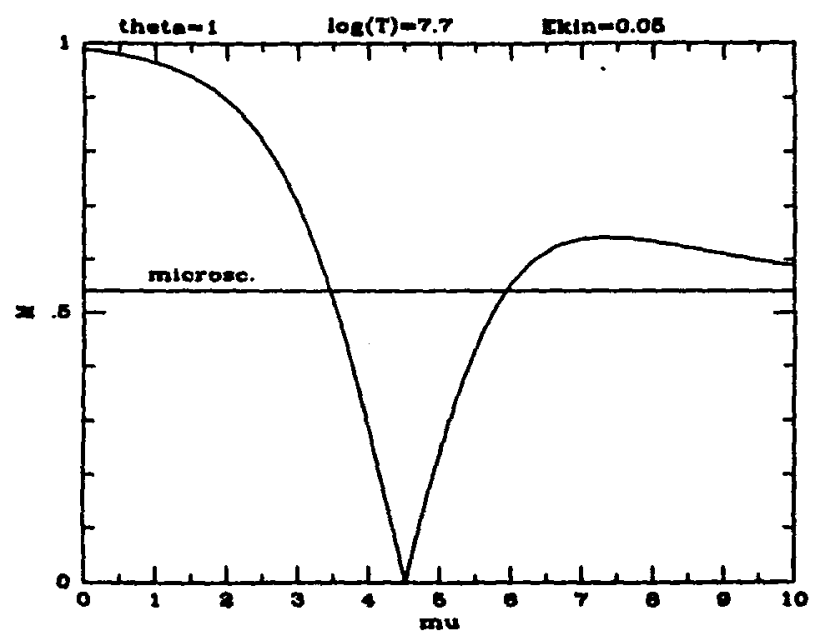

Fig. 33.1 The macroscopic magnetization $M$ normalized upon one is plotted versus chemical potential for $T=5 * 10^{7} \mathrm{~K}$ and electron energy $E_{k i n}=$ $0.05 * m_{e} * c^{2}$. The microscopic magnetization $M=0.54$. The functional dependence below the critical point is well known, whereas the Maxwellian limit is approached above the Curie point.

condensation within the momentum space at $T=0 K$ and total depolarization of the photon gas at $T \rightarrow \infty$ (Engelhardt, Bues 1992). The properties of the local Fermi function are shown in Fig.1-2, where the magnetization $M$, and the critical index $\gamma$, defined by $M=\left\langle E * \rho * \sigma>\propto\left|T-T_{c}\right|^{\gamma}\right.$, with $T_{c}$ the Curie temperature and $\sigma$ one Dirac matrix, are plotted. The critical index is similiar to solid state models and measurements. Therefore the model should provide a description of magnetized Fe.

\subsection{The white dwarf model}

The global white dwarf model is constructed by the use of the standard white dwarf theory (Lieb 1987) and the matrix Fermi function. First we set up the equation of state, then a density model is constructed and the effect of the energy of the magnetic field upon the structure of the white dwarf is discussed.

The pressure must be defined consistently with respect to Lorentz rotations and translatorial transformations. The pressure $P$ is related to the energy, e.g.:

$$
P=\langle E\rangle / V=\frac{1}{(2 \pi \hbar)^{3}} \int d^{3} k \frac{E \rho}{\exp (\beta H)+1} .
$$




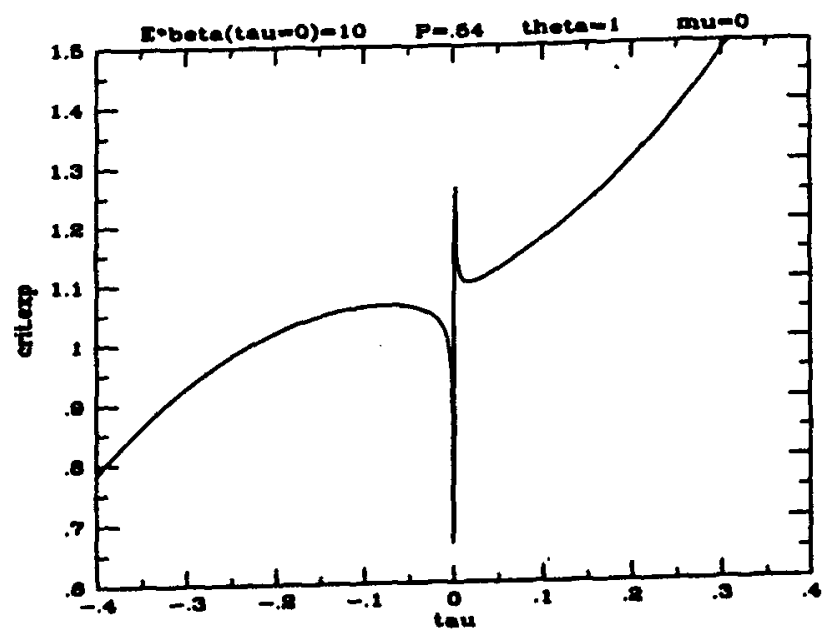

Fig. 33.2 The critical exponent $\gamma$ is plotted versus $\tau=\left(T-T_{c}\right) / T_{c}$, where $T_{r}$ is the Curie temperature, for a microscopic polarization $M=0.54$. The relation of kinetic energy per thermal energy is 10 at the Curie point. The chemical potential $m u$ is equal to zero. The critical exponent at $M=0$ equals approximately 1.1 , this ayrees with measurements of solid state.

The macroscopic density is defined as

$$
n=\frac{1}{(2 \pi \hbar)^{3}} \int d^{3} k \frac{1}{\exp (\beta H)+1},
$$

where $\hbar$ is Planck's constant, $\mathrm{k}$ is the momentum and $\mathrm{V}$ is the volume. The eigen-values of $E * \rho$ reflect the linear or quadratic energy/momentum relation and the isochronic Lorentz group. The interaction energy or the chemical potential relates via the Boltzmann distribution equation (7) and (6). The pressure in terms of the Maxwellian limit of the Fermi function has the form of a Lorentz force, e.g.:

$$
P \propto \int d^{3} k E * \rho
$$

which leads to the usual polytropic index $\gamma^{\prime}=4 / 3 . .5 / 3$ dependent on the relativistic energy. The behaviour of electron crystallization is more complex, because the phase transition depends on temperature and the electrons within the magnetized crystal interact with each other non locally in space.

The nonlocal model assumes, that all states of the crystal are filled up according to Pauli's principle due to the gravitation of the crystal itself until a relativistic Fermi momentum. A constant temperature of $5 * 10^{7} \mathrm{~K}$ inside the crystal is assumed. The number of states are counted consistently with the chemical potential.

The behaviour of the total equilibrium of the star is considered in terms 


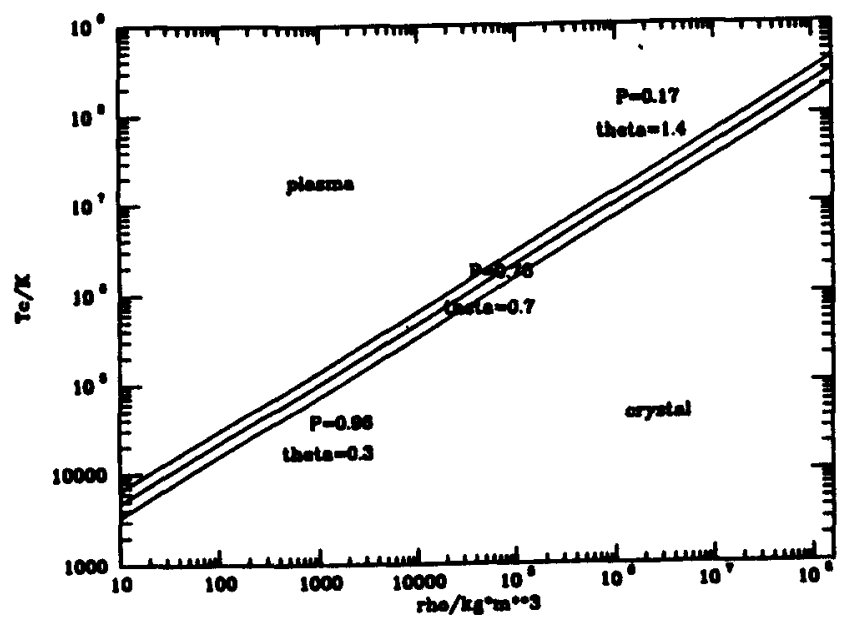

Fig. 33.3 The phase transition from the Maxwellian plasma to the magnetized crystal is plotted in terms of density and Curie temperature for different microscopic magnetizations $P=0.96, P=0.76, P=0.17$.

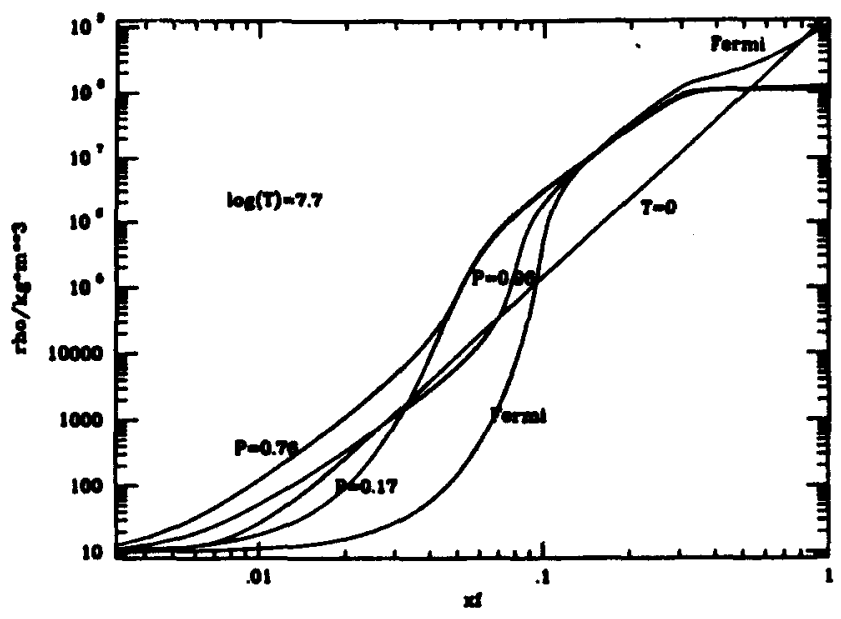

Fig. 33.4 The density versus Fermi momentum in units if $m_{\epsilon} * c^{2}$ for different polarizations $P=0.17,0.76,0.96$ of the Plasma. The non polarized Fermi density as well as the zero temperature density is shown. The model is valid for $x f>0.1$. It is remarkable, that the maximum polarization curve approaches the usual scalar Fermi function inside the crystal. The central density of the crystal approaches $10^{8} \mathrm{~kg} / \mathrm{m}^{3}$.

of the virial theorem. The gravitational energy is balanced by the pressure in matrix form. The additional pressure dependent on the magnetic field is written according to Cox et al. (1968) in terms of the gravitational energy with magnetic flux conservation inside and outside of the crystal. 


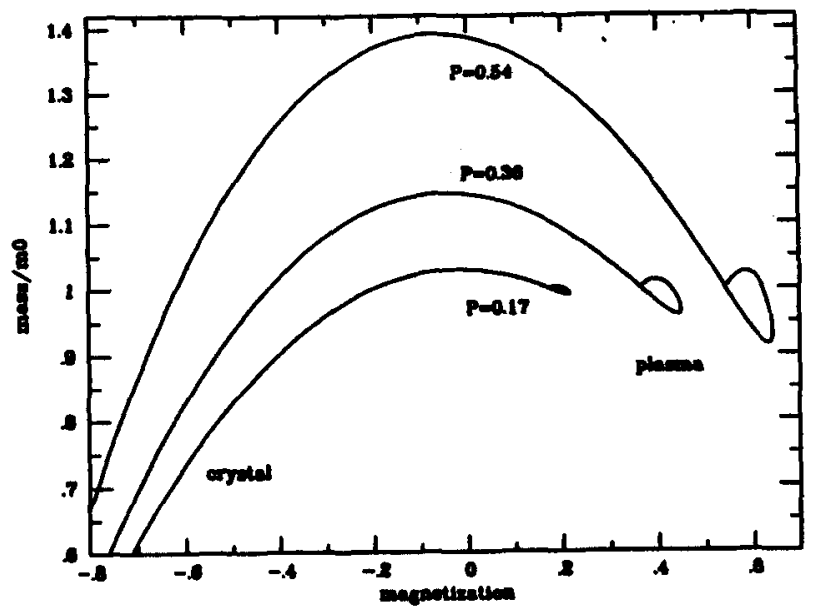

Fig. 33.5 The mass in units of $1.44 M_{0}$ versus macroscopic magnetization $M$ for different polarizations $P=0.17,0.36,0.54$ of the nondegenerate plasma. The phase transition enhances the pressure at $M=0$, whereas the binding energy of the crystal is lower than the scalar Fermi energy.

This picture avoids the singularity which occurs, when the pressure density relation is written in a polytropic form.

\subsection{Conclusion}

From the analysis within the combined Chandrasekhar - Heisenberg model we conclude, that a white dwarf with a magnetic field, which is beginning to crystallize has a larger Chandrasekhar mass of $1.4 * 1.44 M_{0}$ for the plasma regime above the crystallization zone which is polarized at half maximum. Along the cooling sequence a larger magnetic field is needed to stabilize the white dwarf.

\section{References}

Cox, J.P., Giuli, R.T., stellar structure, Science, New York, (1968)

Engelhardt, D., Bues, I., White Duarfs, Kluwer, Dordrecht, 229, (1993)

Grosse,H., Models in Statistical Physics and Quantum Field, Springer,Berlin, (1988)

Jones, R.C., J.Opt.Soc. Am., 31,488, (1941)

Lieb,E.H., Commun.Muth.Phys.,112, 147, (1987)

Sakurai J.J., Advanced Quantum Mechanics, Addison- Wesley, Reading, (1967) 